\title{
Protection of liposomes against fusion during drying by oligosaccharides is not predicted by the calorimetric glass transition temperatures of the dry sugars
}

\author{
Dirk K. Hincha $\cdot$ Petra Rennecke • Ann E. Oliver
}

Received: 7 September 2007 / Revised: 14 November 2007 / Accepted: 20 November 2007 / Published online: 7 December 2007

(C) The Author(s) 2007

\begin{abstract}
Sugars play an important role in the desiccation tolerance of most anhydrobiotic organisms. It has been shown in previous studies that different structural families of oligosaccharides have different efficacies to interact with phospholipid headgroups and protect membranes from solute leakage during drying. Here, we have compared three families of linear oligosaccharides (fructans (inulins), malto-oligosaccharides, manno-oligosaccharides) for their chain-length dependent protection of egg phosphatidylcholine liposomes against membrane fusion. We found increased protection with chain length up to a degree of polymerization (DP) of 5 for malto-oligosaccharides, and a decrease for inulins and manno-oligosaccharides. Differential scanning calorimetry measurements showed that for all sugars the glass transition temperature $\left(T_{\mathrm{g}}\right)$ increased with DP, although to different degrees for the different oligosaccharide families. Higher $T_{\mathrm{g}}$ values resulted in reduced membrane fusion only for malto-oligosaccharides below DP5. Contrary to expectation, for inulins, manno-oligosaccharides and malto-oligosaccharides of a DP above five,
\end{abstract}

\section{K. Hincha}

Max-Planck-Institut für Molekulare Pflanzenphysiologie,

Am Mühlenberg 1, 14476 Potsdam, Germany

\section{P. Rennecke}

School of Veterinary Medicine,

University of California, Davis, CA 95616, USA

A. E. Oliver

Department of Applied Sciences,

University of California, Davis, CA 95616, USA

D. K. Hincha ( $\square)$

Max-Planck-Institut für Molekulare Pflanzenphysiologie,

Am Mühlenberg 1, 14424 Potsdam, Germany

e-mail: hincha@mpimp-golm.mpg.de fusion increased with increasing $T_{\mathrm{g}}$, indicating that other physical parameters are more important in determining the ability of different sugars to protect membranes against fusion during drying. Further research will be necessary to experimentally define such parameters.
Abbreviations
DP Degree of polymerization
EPC Egg phosphatidylcholine
Fru Fructose
Gal Galactose
Glc Glucose
Man Mannose
NBD-PE $\quad N$-(7-nitro-2,1,3-benzoxadiazol-4-yl) phosphatidylethanolamine
RH Relative humidity
Rh-PE $\quad N$-(lissamine rhodamine B sulfonyl) dioleoyl-phosphatidylethanolamine
RFO Raffinose-family oligosaccharides
Suc Sucrose
$T_{\mathrm{c}} \quad$ Critical temperature
TEN TES-EDTA-NaCl buffer
$\mathrm{Tg} \quad$ Calorimetric glass transition temperature

\section{Introduction}

Sugars have the ability to stabilize biological cells during desiccation (see Crowe et al. 1992 for a review). In this regard, the effects of sugars on the stability of liposomes, which are a convenient model of biological membranes, during drying have been extensively investigated (see Hincha et al. 2006 for a recent review). Much of this research has focused on the disaccharides Suc and trehalose, while other potential protectants, such as oligosaccharides, have 
received less attention (see Oliver et al. 2002, 2001 for reviews).

It has, however, been shown that manno-oligosaccharides (linear oligomers of $\beta 1-4$ linked Man; Man- $\operatorname{Man}_{n}$; with $n=$ number of monosaccharide units) (Cacela and Hincha 2006b) and malto-oligosaccharides (linear oligomers of $\alpha 1-4$ linked Glc; Glc-Glc ${ }_{n}$ ) (Cacela and Hincha 2006b; Hincha et al. 2002; Suzuki et al. 1996) decrease in their effectiveness to protect liposomes from leakage of a soluble marker during drying with increasing DP. RFO (linear oligomers of Gal $\alpha 1-6$ linked with a capping Suc; Glc-Fru-Gal ${ }_{n}$ ) (Hincha et al. 2003) and inulins (linear oligomers of Fru $\beta 2-1$ linked with a capping Glc; Glc-Fru ${ }_{n}$ ) (Cacela and Hincha 2006b; Hincha et al. 2002), on the other hand, become more effective with increasing DP. Recently, it has been shown that the branched fructans from cereals such as oat and rye show a more complex dependence of membrane stabilization on DP, with optima at intermediate chain lengths (Hincha et al. 2007).

The differences between the different linear oligosaccharides have been related to their ability to interact with the lipid headgroups in the dry state. In particular, differences in the extent of $\mathrm{H}$-bonding of different oligo-saccharides of the same DP to the $\mathrm{P}=\mathrm{O}$ groups (Cacela and Hincha 2006a, b; Hincha et al. 2002, 2003) have been related to the differential protection of liposomes from leakage during drying. Similar differences have been reported for interactions with the more exposed choline group of EPC, but not for the more buried $\mathrm{C}=\mathrm{O}$ group (Cacela and Hincha 2006b). Such H-bonding interactions of sugar OH-groups with the lipid headgroups are thought to replace hydration water of the lipids during drying (Oliver et al. 1998a) and are closely related to the ability of different sugars to depress the lipid liquid-crystalline to gel phase transition temperature in the dry state, similar to the effect of water (Cacela and Hincha 2006a).

Another important determinant of membrane stability during drying is the prevention of membrane fusion between liposomes, which can also lead to solute leakage. It is generally accepted that sugars prevent liposome fusion during drying by forming a glass (vitrification) and thereby preventing the close approach of vesicles necessary for fusion, without the need for specific interactions with the membranes (see Buitink and Leprince 2004; Crowe et al. 1998 for reviews). The ability of a substance to vitrify can be experimentally characterized by its $T_{\mathrm{g}}$. In oligo- and polymers, $T_{\mathrm{g}}$ generally increases with molecular weight (Slade and Levine 1991) and an increase in $T_{\mathrm{g}}$ with DP has been reported for RFO (Buitink et al. 2000a), fructans (Hinrichs et al. 2001; Schaller-Povolny et al. 2000), and malto-oligosaccharides (Orford et al. 1989). However, while RFO and malto-oligosaccharides induced a decrease in membrane fusion with increasing DP (Hincha et al. 2002, 2003), fusion increased with DP in the presence of inulins (Hincha et al. 2002). These effects on fusion are notably different from the effects on leakage and the ability of the oligosaccharides to $\mathrm{H}$-bond to lipid headgroups, indicating that different properties of the sugars may be responsible for their effects on leakage and fusion.

In the present paper we report a systematic study of the effect of DP on the ability of inulins, malto-oligosaccharides and manno-oligosaccharides to prevent liposome fusion during drying and compare these data with the $T_{\mathrm{g}}$ values of the respective sugars. Surprisingly, our analysis shows that while $T_{\mathrm{g}}$ increased with DP in all three oligosaccharide families, membrane fusion decreased only in the presence of malto-oligosaccharides up to DP5. In the presence of inulins, manno-oligosaccharides and higher DP malto-oligosaccharides fusion increased both as a function of DP and $T_{\mathrm{g}}$.

\section{Materials and methods}

\section{Materials}

EPC was purchased from Lipid Products (South Nutfield, Surrey, UK). NBD and Rh-PE were obtained from Molecular Probes (Leiden, The Netherlands). Suc and malto-oligosaccharides were obtained from Sigma, inulins and mannooligosaccharides were purchased from Megazyme (Wicklow, Ireland). The purity of the sugars was at least $95 \%$, as determined by analytical HPLC, as described in detail in previous publications (Hincha et al. 2002, 2003).

\section{Preparation of liposomes}

Lipids were dried from chloroform under a stream of $\mathrm{N}_{2}$ and stored under vacuum overnight to remove traces of solvent. Liposomes were prepared from lipids hydrated in TEN buffer (10 mM TES, $0.1 \mathrm{mM}$ EDTA (pH 7.4), $50 \mathrm{mM}$ $\mathrm{NaCl}$ ), using a hand-held extruder with two layers of polycarbonate membranes with $100 \mathrm{~nm}$ pores (MacDonald et al. 1991; Avestin, Ottawa, Canada).

\section{Membrane fusion experiments}

Liposome fusion after drying and rehydration was determined using fluorescence resonance energy transfer (Struck et al. 1981) as described in detail before (Hincha et al. 1998). Briefly, two EPC liposome samples were prepared: one sample was labeled with $1 \mathrm{~mol} \%$ each of the fluorescent probe pair NBD-PE and Rh-PE, while the other sample was unlabeled. The two samples were combined after extrusion in TEN buffer in a 1:9 (labeled:unlabeled) ratio, resulting in a final lipid concentration of $10 \mathrm{mg} / \mathrm{ml}$. 
Liposomes $(40 \mu \mathrm{l})$ were mixed with an equal volume of concentrated solutions of sugars in TEN and $20 \mu 1$ aliquots were filled into the caps of microcentrifuge tubes. The samples were dried in desiccators at $28^{\circ} \mathrm{C}$ and $>1 \% \mathrm{RH}$ for $24 \mathrm{~h}$ in the dark (Hincha et al. 2002). Fusion was measured after rehydration in TEN buffer by fluorescence resonance energy transfer (Struck et al. 1981) with a Kontron SFM 25 fluorometer (Kontron Instruments, Neufahrn, Germany) at excitation and emission wavelengths of 450 and $530 \mathrm{~nm}$, respectively. Due to a slight quenching effect of Triton-X 100 on NBD fluorescence, fusion values above $100 \%$ are calculated (Fig. 1) for fully fused liposomes (see Tomczak et al. 2001 for details).

\section{Differential scanning calorimetry of sugar glasses}

Glass transitions $\left(T_{\mathrm{g}}\right)$ were measured using a Perkin Elmer DSC-7 (Norwalk, CT, USA) or a Mettler-Toledo DSC822 differential scanning calorimeter (Columbus, OH, USA), both equipped for subambient operation. Sugar solutions were dissolved in $18 \mathrm{M} \Omega$ water at $200-500 \mathrm{mg} / \mathrm{ml}$ and air dried into Differential scanning calorimetry (DSC) pans in a sealed box that was continuously purged with dry air of less than $3 \%$ RH over a period of 2-3 days. Finally, samples were incubated for $\sim 1 \mathrm{~h}$ at elevated temperature $\left(60-80^{\circ} \mathrm{C}\right)$ to remove residual water and pressure-sealed in the dry box. For the state diagram, samples were transferred to chambers maintained at specific RH over saturated salt solutions (Weast 1984) for 1-24 h before sealing. Samples were scanned in the range of -50 to $200^{\circ} \mathrm{C}$ at $10^{\circ} \mathrm{C} / \mathrm{min}$. Glass transitions were analyzed using the Perkin Elmer or Mettler software, which determine $T_{\mathrm{g}}$ as the stable midpoint of the baseline inflection. Every experiment was performed twice, with at least three heating runs for each experiment. Water contents were determined gravimetrically using a Cahn C-33 electrobalance, as previously described (Oliver et al. 1998b).

\section{Results and discussion}

We have investigated the effects of three structural families of oligosaccharides, inulins, malto-oligosaccharides, and manno-oligosaccharides, on membrane fusion in the dry state. While fusion data have been reported previously for the first two families (Hincha et al. 2002), the last has not
Fig. 1 Protection of large unilamellar liposomes from damage during drying by inulins, maltooligosaccharides and mannooligosaccharides of different degrees of polymerization $(D P)$. Fusion of the vesicles after airdrying and rehydration is shown as a function of the concentration of the sugars in the suspending medium. Data points represent means \pm SEM from three parallel samples. The data for inulins and malto-oligosaccharides were taken from Hincha et al. (2002)

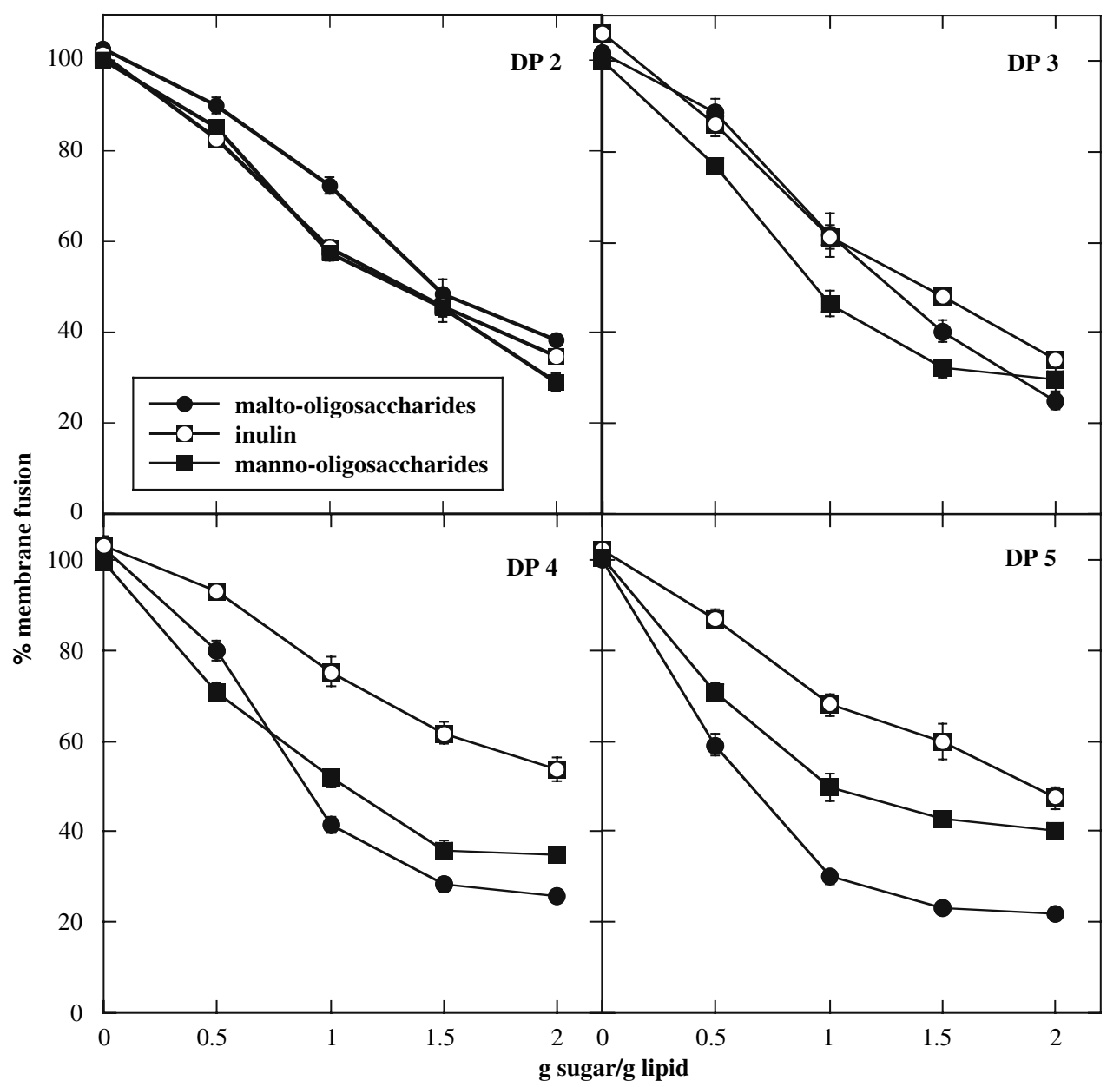


been investigated previously. We used commercially available sugars from DP2 to DP5 for inulins and manno-oligosaccharides, and DP2 to DP7 for malto-oligosaccharides.

Figure 1 shows the effects of oligosaccharides up to DP5 on liposome fusion after drying and rehydration. All sugars showed concentration dependent protection of liposomes against fusion. The degree of protection, however, varied with DP and oligosaccharide family. While the malto-oligosaccharides showed increased protection up to DP5, the members of the other two families showed a linear decrease in protection with increasing DP (Figs. 1, 2). However, at higher DP (DP6 and 7), the effectiveness of the malto-oligosaccharides also decreased (Fig. 2). These experiments have identified, in addition to data reported before for inulins and malto-oligosaccharides (Hincha et al. 2002), manno-oligosaccharides as another family of sugars that shows less inhibition of fusion with increasing DP. The effect of DP was not as strong as for the inulins, but clearly in the opposite direction than for the malto-oligosaccharides up to DP5 (Figs. 1, 2).

The basic assumption about the role of sugars in preventing membrane fusion during drying is that it is due to the formation of a glass phase (see Crowe et al. 1998; Hincha et al. 2006 for reviews). In addition, it has been shown that heating samples containing Suc above the sugar glass tran-

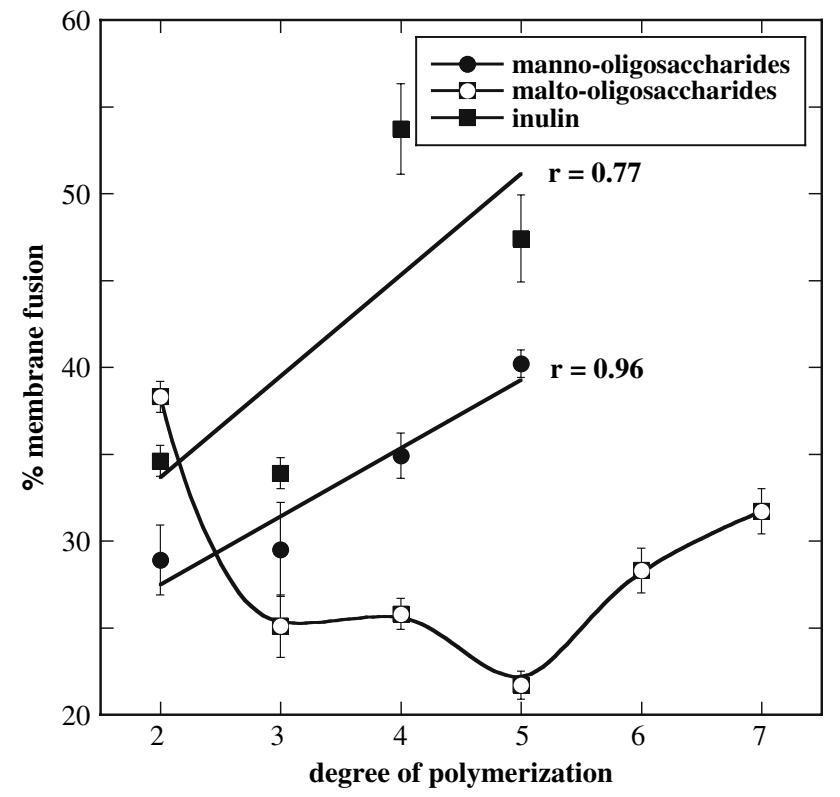

Fig. 2 Percentage of liposome fusion after air-drying and rehydration as a function of the DP of inulins, malto-oligosaccharides and mannooligosaccharides. All samples contained $2 \mathrm{~g}$ sugar/g lipid (compare Fig. 1). The straight lines for samples containing inulins and mannooligosaccharides were fitted by least-squares linear regression. The regression coefficients $(r)$ are shown next to the respective lines. The line connecting the data from the malto-oligosaccharide containing samples is only meant as a visual aid. The data for inulins and maltooligosaccharides were taken from Hincha et al. (2002) sition temperature leads to a rapid increase in membrane damage (Sun et al. 1996). Therefore, we compared the vitrification behavior of the oligosaccharides by DSC to elucidate the interdependence of membrane fusion, DP, and $T_{\mathrm{g}}$.

Figure 3 shows state diagrams for the glass transitions of manno-oligosaccharides, malto-oligosaccharides and inulins of DP5. In common with other sugar glasses (Buitink and Leprince 2004; Crowe et al. 1998), water acted as a plasticizer and led to a progressive decrease in $T_{\mathrm{g}}$ with increasing water content. The degree of reduction in $T_{\mathrm{g}}$, however, was different for the different oligosaccharides, in accordance with findings for other oligo- and polysaccharides (Sun et al. 2004). It was not possible to obtain a $T_{\mathrm{g}}$ value for extremely dry (below $0.13 \mathrm{~g}$ water/g dry weight) DP5 manno-oligosaccharide, because the sample decomposed during heating before $T_{\mathrm{g}}$ was reached. However, the data suggest that at a given water content the DP5 inulin showed the lowest and the corresponding manno-oligosaccharide the highest $T_{\mathrm{g}}$, with the malto-oligosaccharide intermediate.

For all three families of oligosaccharides, $T_{\mathrm{g}}$ increased with DP, as expected (Fig. 4a). The increase was highest for the malto-oligosaccharides and lowest for the inulins, with the manno-oligosaccharides intermediate. The caveat in the case of the manno-oligosaccharides is; however, that in the DP5 sample, water content was higher than in all other samples (compare also Fig. 3), so that $T_{\mathrm{g}}$ may be underestimated in this case. Similarly, $T_{\mathrm{g}}$ was slightly higher for the DP2 than for the DP3 manno-oligosaccharide, indicating that its water content was lower.

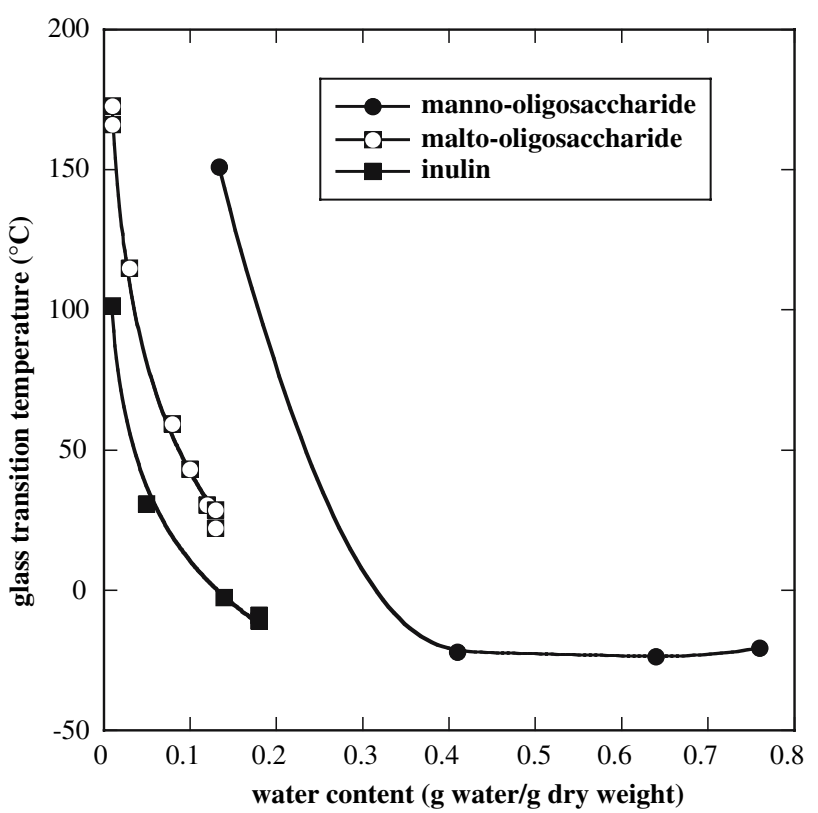

Fig. 3 State diagram of DP5 sugars of different oligosaccharide families as a function of water content in the samples 
Unfortunately, the sugar in this sample decomposed during gravimetric analysis, so that no exact water content data are available. However, a sample of DP2 manno-oligosaccharide with a higher-water content $(0.26 \mathrm{~g}$ water/g dry weight) showed the expected lower $T_{\mathrm{g}}\left(27.4^{\circ} \mathrm{C}\right)$. In addition, double reciprocal plots (Fig. 4b) yielded the expected straight lines for all oligosaccharide families (Orford et al. 1989), indicating that the data reflect the relative vitrification behavior of the sugars reasonably well.
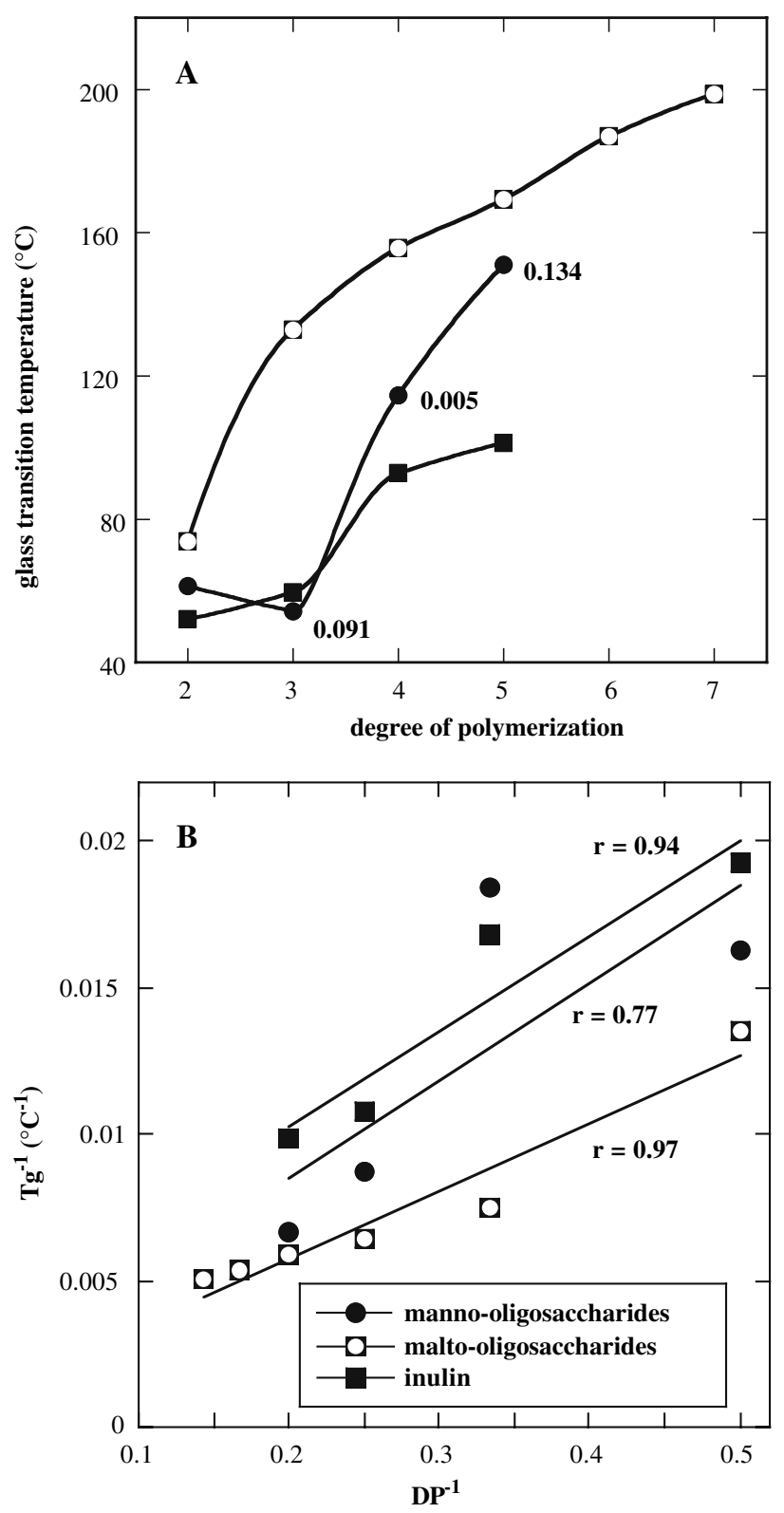

Fig. 4 Glass transition temperatures of sugars from the different oligosaccharide families as a function of $\operatorname{DP}(\mathbf{a})$. Panel (b) shows a double reciprocal plot of the data in $\mathbf{a}$. The water content of the samples was below $0.01 \mathrm{~g}$ water/g dry weight for all inulins and malto-oligosaccharides, but is indicated in the figure for the manno-oligosaccharides of DP3-DP5 (see text for further details)
A plot of membrane fusion as a function of the $T_{\mathrm{g}}$ of the different sugars presents a surprising picture (Fig. 5). Only malto-oligosaccharides up to DP5 show the expected behavior, i.e. a reduction in fusion with increasing DP. The higher DP malto-oligosaccharides and all other oligosaccharides, on the contrary, show an increase in membrane fusion with increasing $T_{\mathrm{g}}$. On the other hand, it has been shown before that sorbitol is a much better protectant against membrane fusion during drying than would be expected from its $T_{\mathrm{g}}$ (Hincha and Hagemann 2004). It has been suggested previously that $T_{\mathrm{c}}$, the critical temperature where a glass changes from the solid-like to the liquid-like state, may be a better parameter for the functional characterization of sugar glasses (Buitink et al. 2000b). $T_{\mathrm{c}}$ for sorbitol, for example, is approximately $40^{\circ} \mathrm{C}$ above $T_{\mathrm{g}}$ (Tsujimi et al. $1999 \mathrm{a}, \mathrm{b}$ ), while it is only $18^{\circ} \mathrm{C}$ above $T_{\mathrm{g}}$ for sucrose (Buitink et al. 2000b). Unfortunately, no $T_{\mathrm{c}}$ data for the oligosaccharides investigated in our study have been published.

In addition, it has been shown in recent spectroscopic investigations that there is spatial and temporal heterogeneity in sugar glasses, even at temperatures considerably below $T_{\mathrm{g}}$ and that this heterogeneity is different for different sugars and sugar alcohols (Shirke and Ludescher 2005a, b).

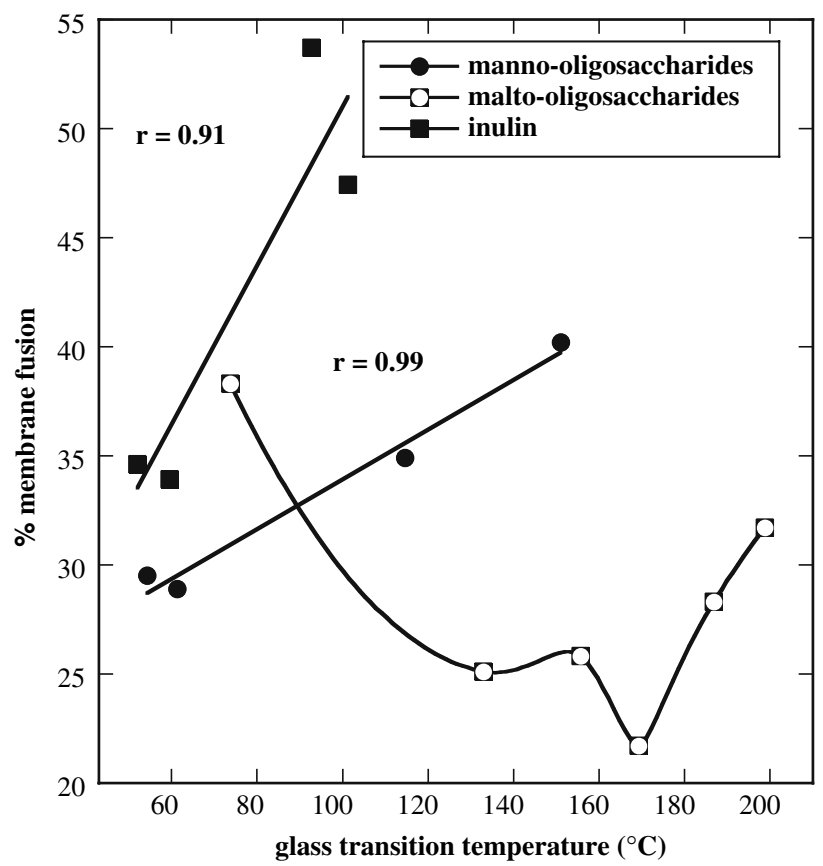

Fig. 5 Percentage of liposome fusion after air-drying and rehydration as a function of the glass transition temperature of the respective oligosaccharides of different DP. The fusion data are taken from samples that contained $2 \mathrm{~g}$ sugar/g lipid (compare Figs. 1, 2). The straight lines for samples containing inulins and manno-oligosaccharides were fitted by least-squares linear regression. The regression coefficients $(r)$ are shown next to the respective lines. The line connecting the data from the malto-oligosaccharide containing samples is only meant as a visual aid 


\section{Conclusions}

While there is little doubt that vitrification of the sugar matrix during drying is necessary to prevent membrane fusion (Buitink and Leprince 2004; Crowe et al. 1998) and that fusion is increased when samples are heated above $T_{\mathrm{g}}$ (Sun et al. 1996), the present study shows that measurements of $T_{\mathrm{g}}$ are not a good predictor of membrane protection against fusion for different sugars. This is even true for oligosaccharides of the same DP, so that effects of size differences can be largely excluded. Therefore, our data indicate that structural differences between different sugars play an important role not only in the ability of sugars to interact with lipid headgroups (Cacela and Hincha 2006b), but also in their ability to form a glass that effectively prevents membrane fusion. Further investigations will be necessary to relate structural differences between different sugars to functional differences, to understand the structural basis of the protective abilities of different oligosaccharides.

Open Access This article is distributed under the terms of the Creative Commons Attribution Noncommercial License which permits any noncommercial use, distribution, and reproduction in any medium, provided the original author(s) and source are credited.

\section{References}

Buitink J, Leprince O (2004) Glass formation in plant anhydrobiotes: survival in the dry state. Cryobiology 48:215-228

Buitink J, Hemminga MA, Hoekstra FA (2000a) Is there a role for oligosaccharides in seed longevity? An assessment of intracellular glass stability. Plant Physiol 122:1217-1224

Buitink J, van den Dries IJ, Hoekstra FA, Alberda M, Hemminga MA (2000b) High critical temperature above $T_{\mathrm{g}}$ may contribute to the stability of biological systems. Biophys J 79:1119-1128

Cacela C, Hincha DK (2006a) Low amounts of sucrose are sufficient to depress the phase transition temperature of dry phosphatidylcholine, but not for lyoprotection of liposomes. Biophys J 90:2831-2842

Cacela C, Hincha DK (2006b) Monosaccharide composition, chain length and linkage type influence the interactions of oligosaccharides with dry phosphatidylcholine membranes. Biochim Biophys Acta 1758:680-691

Crowe JH, Hoekstra FA, Crowe LM (1992) Anhydrobiosis. Annu Rev Physiol 54:579-599

Crowe JH, Carpenter JF, Crowe LM (1998) The role of vitrification in anhydrobiosis. Annu Rev Physiol 60:73-103

Hincha DK, Hagemann M (2004) Stabilization of model membranes during drying by compatible solutes involved in the stress tolerance of plants and microorganisms. Biochem J 383:277-283

Hincha DK, Oliver AE, Crowe JH (1998) The effects of chloroplast lipids on the stability of liposomes during freezing and drying. Biochim Biophys Acta 1368:150-160

Hincha DK, Zuther E, Hellwege EM, Heyer AG (2002) Specific effects of fructo- and gluco-oligosaccharides in the preservation of liposomes during drying. Glycobiology 12:103-110

Hincha DK, Zuther E, Heyer AG (2003) The preservation of liposomes by raffinose family oligosaccharides during drying is mediated by effects on fusion and lipid phase transitions. Biochim Biophys Acta 1612:172-177

Hincha DK, Popova AV, Cacela C (2006) Effects of sugars on the stability of lipid membranes during drying. In: Leitmannova Liu A (ed) Advances in planar lipid bilayers and liposomes, vol 3. Elsevier, Amsterdam, pp 189-217

Hincha DK, Livingston DP III, Premakumar R, Zuther E, Obel N, Cacela C, Heyer AG (2007) Fructans from oat and rye: composition and effects on membrane stability during drying. Biochim Biophys Acta 1768:1611-1619

Hinrichs WLJ, Prinsen MG, Frijlink HW (2001) Inulin glasses for the stabilization of therapeutic proteins. Int J Pharm 215:163-174

MacDonald RC, MacDonald RI, Menco BPM, Takeshita K, Subbarao NK, Hu L (1991) Small-volume extrusion apparatus for preparation of large, unilamellar vesicles. Biochim Biophys Acta 1061:297-303

Oliver AE, Crowe LM, Crowe JH (1998a) Methods for dehydration tolerance: depression of the phase transition temperature in dry membranes and carbohydrate vitrification. Seed Sci Res 8:211221

Oliver AE, Hincha DK, Crowe JH (2002) Looking beyond sugars: the role of amphiphilic solutes in preventing adventitious reactions in anhydrobiotes at low water contents. Comp Biochem Physiol 131A:515-525

Oliver AE, Hincha DK, Crowe LM, Crowe JH (1998b) Interactions of arbutin with dry and hydrated bilayers. Biochim Biophys Acta 1370:87-97

Oliver AE, Leprince O, Wolkers WF, Hincha DK, Heyer AG, Crowe JH (2001) Non-disaccharide-based mechanisms of protection during drying. Cryobiology 43:151-167

Orford PD, Parker R, Ring SG, Smith AC (1989) Effect of water as a diluent on the glass transition behaviour of malto-oligosaccharides, amylose and amylopectin. Int J Biol Macromol 11:91-96

Schaller-Povolny LA, Smith DE, Labuza TP (2000) Effect of water content and molecular weight on the moisture isotherms and glass transition properties of inulin. Int J Food Properties 3:173-192

Shirke S, Ludescher RD (2005a) Dynamic site heterogeneity in amorphous maltose and maltitol from spectral heterogeneity in erythrosin B phosphorescence. Carbohydr Res 340:2661-2669

Shirke S, Ludescher RD (2005b) Molecular mobility and the glass transition in amorphous glucose, maltose, and maltotriose. Carbohydr Res 340:2654-2660

Slade L, Levine H (1991) Beyond water activity: recent advances based on an alternative approach to the assessment of food quality and safety. Crit Rev Food Sci Technol 30:115-360

Struck DK, Hoekstra D, Pagano RE (1981) Use of resonance energy transfer to monitor membrane fusion. Biochemistry 20:4093-4099

Sun WQ, Leopold AC, Crowe LM, Crowe JH (1996) Stability of dry liposomes in sugar glasses. Biophys J 70:1769-1776

Sun WQ, Wagner CT, Connor J (2004) The glass transition behaviors of hydroxyethyl starch solutions. Cell Preserv Technol 2:55-65

Suzuki T, Komatsu H, Miyajima K (1996) Effects of glucose and its oligomers on the stability of freeze-dried liposomes. Biochim Biophys Acta 1278:176-182

Tomczak MM, Hincha DK, Estrada SD, Feeney RE, Crowe JH (2001) Antifreeze proteins differentially affect model membranes during freezing. Biochim Biophys Acta 1511:255-263

Tsujimi Y, Kobayashi M, Furuta H, Yagi T (1999a) Ultra-slow relaxation dynamics in triglycine sulfate and D-sorbitol studied by time-resolved spectroscopy. J Therm Anal Cal 57:859-865

Tsujimi Y, Kobayashi M, Yagi T (1999b) Frequency and timeresolved spectroscopic study of liquid-glass transitions in D-sorbitol. Physica B 263:310-312

Weast RC (1984) CRC handbook of chemistry and physics, 64 edn. CRC, Boca Raton 